\title{
Hexokinase 2 is dispensable for photoreceptor development but is required for survival during aging and outer retinal stress
}

Eric Weh', Zuzanna Lutrzykowska², Andrew Smith', Heather Hager', Mercy Pawar', Thomas J. Wubben ${ }^{1}$ and Cagri G. Besirli (1)

\begin{abstract}
Photoreceptor death is the ultimate cause of vision loss in many retinal degenerative conditions. Identifying novel therapeutic avenues for prolonging photoreceptor health and function has the potential to improve vision and quality of life for patients suffering from degenerative retinal disorders. Photoreceptors are metabolically unique among other neurons in that they process the majority of their glucose via aerobic glycolysis. One of the main regulators of aerobic glycolysis is hexokinase 2 (HK2). Beyond its enzymatic function of phosphorylating glucose to glucose-6-phosphate, HK2 has additional non-enzymatic roles, including the regulation of apoptotic signaling via AKT signaling. Determining the role of HK2 in photoreceptor homeostasis may identify novel signaling pathways that can be targeted with neuroprotective agents to boost photoreceptor survival during metabolic stress. Here we show that following experimental retinal detachment, p-AKT is upregulated and HK2 translocates to mitochondria. Inhibition of AKT phosphorylation in $661 \mathrm{~W}$ photoreceptor-like cells results in translocation of mitochondrial HK2 to the cytoplasm, increased caspase activity, and decreased cell viability. Rod-photoreceptors lacking HK2 upregulate HK1 and appear to develop normally. Interestingly, we found that HK2-deficient photoreceptors are more susceptible to acute nutrient deprivation in the experimental retinal detachment model. Additionally, HK2 appears to be important for preserving photoreceptors during aging. We show that retinal glucose metabolism is largely unchanged after HK2 deletion, suggesting that the non-enzymatic role of HK2 is important for maintaining photoreceptor health. These results suggest that HK2 expression is critical for preserving photoreceptors during acute nutrient stress and aging. More specifically, p-AKT mediated translocation of HK2 to the mitochondrial surface may be critical for protecting photoreceptors from acute and chronic stress.
\end{abstract}

\section{Introduction}

Nearly 10 million individuals in the US alone are affected with either diabetic retinopathy, age-related macular degeneration, or retinitis pigmentosa, and this number is only predicted to increase due to the aging population of the United States ${ }^{1}$. Although the pathological mechanisms underlying these various retinal

\footnotetext{
Correspondence: Cagri G. Besirli (cbesirli@med.umich.edu)

'Department of Ophthalmology and Visual Sciences, University of Michigan,

1000 Wall St., Ann Arbor, MI 48105, US

${ }^{2}$ University of Michigan, Ann Arbor, MI, US

Edited by N. Bazan
}

degenerations are complex, the ultimate cause of blindness is the death of photoreceptor cells. Therefore, identifying a common mechanism for promoting photoreceptor survival agnostic of the upstream pathological signaling has immense potential for preserving vision in diverse retinal diseases.

Photoreceptors require tremendous amounts of macromolecules to replenish outer segments, which are shed daily $^{2}$. In addition, photoreceptors seem to possess little reserve capacity for energy production and are extremely sensitive to metabolic dysfunction as evidenced by mutations in common metabolism genes leading to 
isolated inherited retinal degenerations. For example, the enzymes inosine monophosphate dehydrogenase 1 (IMPDH1), hexokinase 1 (HK1), and isocitrate dehydrogenase 3 beta (IDH3B) are expressed throughout most tissues in the body; however, mutations affecting these genes result in isolated retinal degeneration ${ }^{3-5}$. These data suggest that photoreceptor metabolism is tightly regulated and small perturbations may lead to deficiencies which cannot be compensated for, resulting in cell death ${ }^{6}$. Identifying pathways important for cell survival during metabolic stress may prove useful for delaying or preventing photoreceptor cell death in many types of retinal degenerations ${ }^{7-10}$. These and other data have led to a hypothesis suggesting that disruption of metabolism and nutrient availability may be a unifying mechanism for photoreceptor death in many degenerative retinal diseases ${ }^{10,11}$.

Photoreceptors utilize a type of metabolism unique among terminally differentiated neurons called aerobic glycolysis, or the Warburg effect, to process the majority of glucose that enters the cell ${ }^{12}$. While most of the glucose entering a cell is normally processed through the citric acid cycle and oxidative phosphorylation, aerobic glycolysis instead diverts pyruvate through lactate dehydrogenase to produce lactate that tends to be exported from the cell. Studies show that $80-90 \%$ of glucose delivered to the outer retina is converted to lactate ${ }^{12-14}$. Photoreceptors may employ this unique metabolic adaptation to meet their high demand for energy and biosynthetic intermediates ${ }^{15}$. Aerobic glycolysis tightly regulates substrate utilization, allowing the shuttling of glycolytic intermediates to other biosynthetic pathways for nucleotide, amino acid, and lipid synthesis or to energy production via oxidative phosphorylation, depending on the physiologic needs of the cell ${ }^{16}$. Three enzymes are key for performing aerobic glycolysis: hexokinase 2 (HK2), pyruvate kinase muscle isozyme 2 (PKM2) and lactate dehydrogenase A (LDHA). We have recently shown that genetically switching PKM2 for PKM1 protects photoreceptors from acute outer retinal stress induced by experimental retinal detachment ${ }^{7}$. Interestingly, HK2 has been shown to play a role in mediating apoptotic cell processes after nutrient stress ${ }^{17,18}$. This identifies HK2 as another attractive target for developing novel neuroprotective interventions in the retina.

Hexokinase 2 (HK2) is one of four main isozymes responsible for the first step in glycolysis. HK2 normally resides on the mitochondria, bound to voltage dependent anion channel (VDAC), where it receives preferential access to ATP for the phosphorylation of glucose to glucose-6-phosphate. While bound to VDAC, HK2 can also prevent the binding of pro-apoptotic factors to the mitochondria and the opening of the mitochondrial permeability transition pore (mPTP), which blocks the release of cytochrome $\mathrm{c}^{18,19}$. Protein kinase B (AKT) has been shown to modulate the ability of HK2 to bind to VDAC through direct phosphorylation ${ }^{20,21}$.

In this report, we show that HK2 preferentially localizes to the mitochondria enriched fraction after outer retinal stress as produced by experimental retinal detachment. The association between HK2 and mitochondria can be partially blocked by inhibiting AKT phosphorylation in vitro, which leads to decreased cell viability and increased caspase activity. We also demonstrate that rod photoreceptor-specific, Hk2 conditional knockout (cKO) mice are more susceptible to acute outer retinal metabolic stress, suggesting an anti-apoptotic role for HK2 during metabolic stress. Additionally, we show that the loss of $H k 2$ in rod photoreceptors does not reprogram metabolism to primarily oxidative phosphorylation. Finally, $H k 2$ cKO mice show significant outer retinal thinning and photoreceptor loss during aging. Collectively, these findings indicate that HK2 is critical for regulating photoreceptor survival during acute metabolic stress and normal aging.

\section{Results \\ HK2 localizes to mitochondria following retinal detachment}

One of the non-enzymatic roles of HK2 is to inhibit apoptosis through its association with mitochondria $^{17,18,20}$. AKT can phosphorylate HK2, which promotes binding to VDAC, an integral mitochondrial outer membrane protein ${ }^{20}$. To determine if this association is important for photoreceptor protection after retinal detachment (RD), HK2 and the ratio of p-AKT/total AKT were assessed following experimental RD in rats (Fig. 1). Three- and 7-days following RD, total HK2 protein expression was decreased significantly (Fig. 1a). Additionally, $H k 2$ transcript levels were significantly decreased at 1- and 3-days post RD (Fig. 1b). Total AKT expression was unchanged, but p-AKT (S473) and the ratio of p-AKT/total AKT was significantly increased (Fig. 1c). To determine if this increase in $\mathrm{p}$-AKT is associated with changes in HK2 sub-cellular localization, rat retinas were detached and harvested at 1-, 3-, and 7-days post RD. After fractionation, HK2 was found to be enriched in the post-cytosolic, mitochondria enriched fraction (hereafter "mitochondrial fraction") 3- and 7-days after RD (Fig. 1d, e), suggesting increased p-AKT may be enhancing HK2 association with mitochondria.

\section{LY294002 treatment induces HK2 translocation to the cytosol and caspase activation}

To better define the relationship between AKT and HK2 localization, $661 \mathrm{~W}$ photoreceptor-like cells were used $^{22,23}$. When treated with LY294002 for $1.5 \mathrm{~h}, \mathrm{HK} 2$ becomes significantly enriched in the cytosolic fraction 


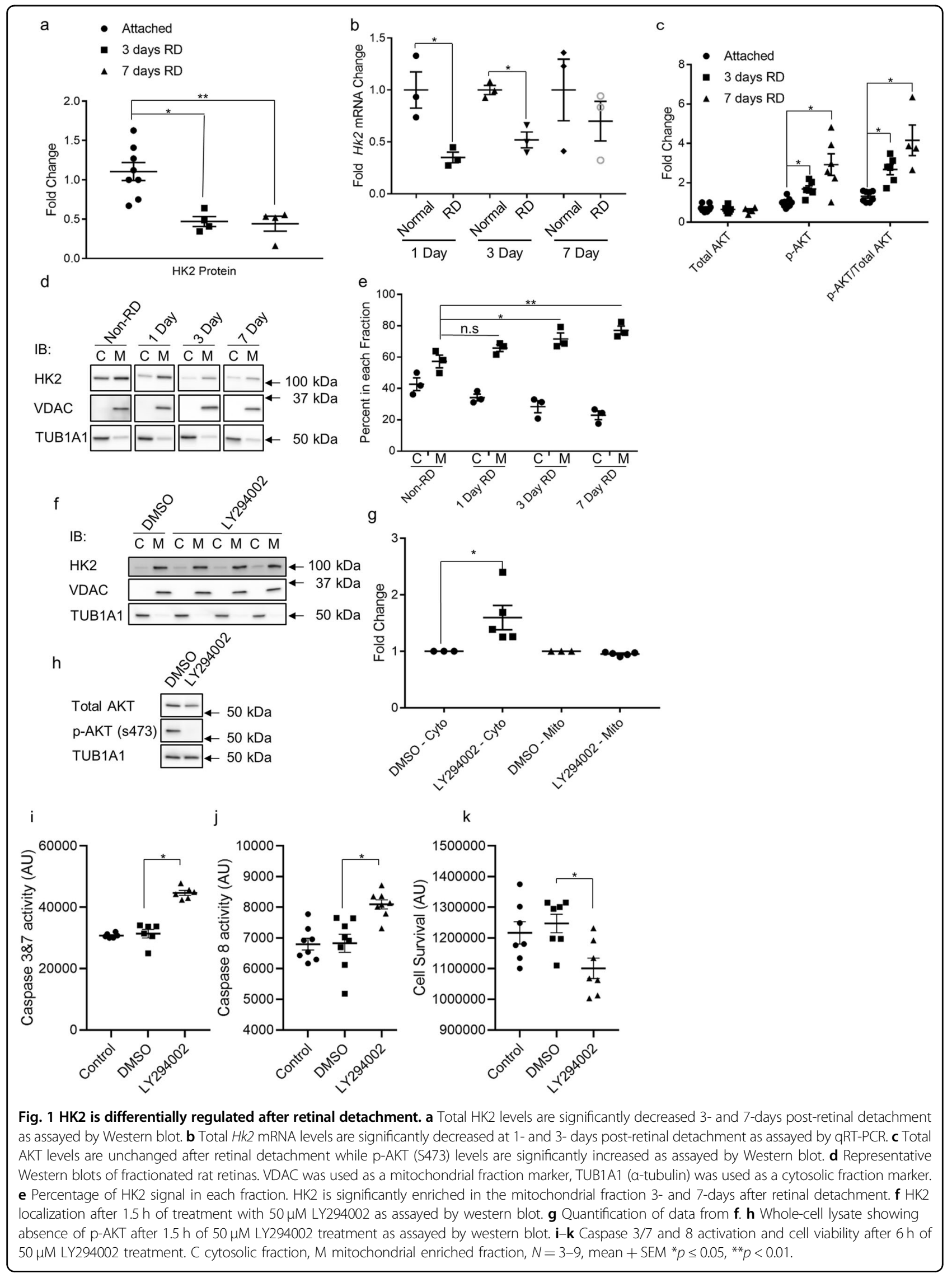


(Fig. 1f, g) and phosphorylation of AKT is nearly abolished (Fig. 1h). After $6 \mathrm{~h}$ of exposure to LY294002, both caspase $3 / 7$ and 8 were significantly activated (Fig. 1i, j) with a corresponding significant decrease in cell viability (Fig. 1k). These data agree with previous studies demonstrating a neuroprotective effect of activated AKT on photoreceptors and suggest that at least part of this neuroprotective effect may be mediated by the regulation of HK2 localization in the cell ${ }^{21,24,25}$.

\section{Deletion of $H k 2$ from rod photoreceptors leads to $H k 1$ upregulation}

These data suggest that HK2 may be important for preserving photoreceptors during apoptotic stress, therefore a rod photoreceptor-specific, Hk2 conditional knockout mouse model was constructed to study $H k 2$ deficiency in photoreceptors ${ }^{26,27}$. Mice with intact $H k 2$ $\left(H k 2^{w t / w t} ; \mathrm{Rho}_{\mathrm{Cr}}{ }^{+}\right.$: WT), and animals lacking $\mathrm{Hk} 2$ in photoreceptors $\left(\mathrm{Hk}^{f l / f l} ; \mathrm{Rho}-\mathrm{Cr} e^{+}\right.$: cKO) were produced. Consistent with other reports, immunofluorescence of retinal sections from WT mice show HK2 is almost exclusively expressed in photoreceptors (Fig. 2a). In cKO animals, HK2 was only present in cone photoreceptors as shown by cone arrestin (ARR3) staining (Fig. 2b) ${ }^{28,29}$. Additionally, WT retinas express low levels of HK1 in photoreceptor inner segments (Fig. 2c) with the majority of HK1 expressed in other cells within the retina. Immunofluorescent staining of retinas from $\mathrm{CKO}$ mice demonstrates that HK1 is upregulated in photoreceptors, predominantly within rod inner segments (Fig. 2c). Western blotting confirmed these results, showing absence of HK2 from cKO retinas while total HK1 levels are significantly increased (Fig. 2d, e). In contrast, total $H k 1$ transcript levels were unchanged (Fig. 2f).

\section{HK2 promotes photoreceptor survival during acute outer retinal stress}

The experimental model of RD utilized here results in increased apoptosis of photoreceptors and one of the main non-enzymatic roles of HK2 is to inhibit apoptosis via its interaction with the mitochondria ${ }^{30-35}$. Furthermore, HK2 was enriched in the mitochondrial fraction after RD in rats (Fig. 1d, e). Therefore, we sought to determine the role of HK2 in photoreceptor survival under acute outer retinal metabolic stress as produced by this experimental model of RD. The retinas of WT and cKO mice were detached and harvested 3 days later, at the peak of TUNEL staining in the outer nuclear layer (ONL) which correlates with long-term photoreceptor survi$\mathrm{val}^{7,36}$. cKO mice had significantly more TUNEL positive cells in the ONL compared to WT mice (Fig. 3a, b). As in our rat model of RD (Fig. 1a), HK2 levels are significantly down-regulated in WT mice 3-days post $\mathrm{RD}$, with no change detected following $\mathrm{RD}$ in $\mathrm{cKO}$ animals (Fig. 3c).
Surprisingly, HK1 protein levels were significantly downregulated in the retinas of cKO mice 3 days after $\mathrm{RD}$, but not in the retinas of WT mice (Fig. 3d). We also determined the localization of HK1 in WT and cKO animals 3-days post RD (Fig. 3e, f). HK1 is significantly enriched in the mitochondrial fraction similar to what is seen with HK2 localization following RD (Fig. 1d, e).

\section{HK2 affects photoreceptor survival and retinal function in aging}

To assess the phenotype resulting from $H k 2$ deletion, in vivo retinal morphology was assessed via optical coherence tomography (OCT). A small but significant decrease in OSEL (outer segment equivalent length) was observed at 1 month but was not present at 5 months of age (Fig. 4a, b). By 10 months of age, a significant decrease in total retinal thickness was observed in cKO mice compared to WT mice (Fig. 4a, b). This thinning is driven by a reduction in ONL and OSEL thickness (Fig. 4b). Eyes from 10-month-old cKO and WT mice were sectioned and stained with hematoxylin and eosin to assess total ONL cell counts. The percent of ONL nuclei was statistically significantly less in cKO compared to WT mice (Fig. 4c, d), consistent with in vivo morphologic assessment. In accordance with these anatomic changes, visual function, as evaluated by electroretinography (ERG), showed no significant changes in any amplitudes at 2 and 5 months of age. Yet, at 10 months of age, statistically significant decreases were noted in the scotopic a-and bwave amplitudes as well as in the photopic b-wave amplitude of cKO animals (Fig. 4e-h).

\section{Deletion of HK2 does not significantly alter retinal metabolism}

Rod photoreceptors have been reported to reprogram their energy metabolism from aerobic glycolysis to oxidative phosphorylation when $H k 2$ is deleted ${ }^{28}$. To investigate this in our $H k 2 \mathrm{cKO}$ model, the transcriptional profile of central glucose metabolism genes was determined in the retina. We conducted real-time PCR using the Mouse Glucose Metabolism $\mathrm{RT}^{2}$ Profiler $^{\mathrm{TM}}$ PCR array (Qiagen), which assesses genes involved in glycolysis, gluconeogenesis, tricarboxylic acid cycle, pentose phosphate pathway, glycogen synthesis, glycogen degradation, and regulation of glucose and glycogen metabolism. Of the 84 genes examined, only seven showed significant $(p<0.05)$ expression differences between the $H k 2 \mathrm{cKO}$ and WT mice (supplemental Table 1, Fig. 5a). Of these, only Fbp2 demonstrated a statistically significant fold change greater than 2. FBP2 (fructose 1,6-bisphosphatase, 2.9-fold change) is the ratelimiting step of gluconeogenesis and catalyzes the conversion of fructose 1,6-bisphosphate to fructose 6-phosphate. Conversion of glucose to lactate is a hallmark of aerobic glycolysis. Photoreceptors process $80-96 \%$ of all glucose 


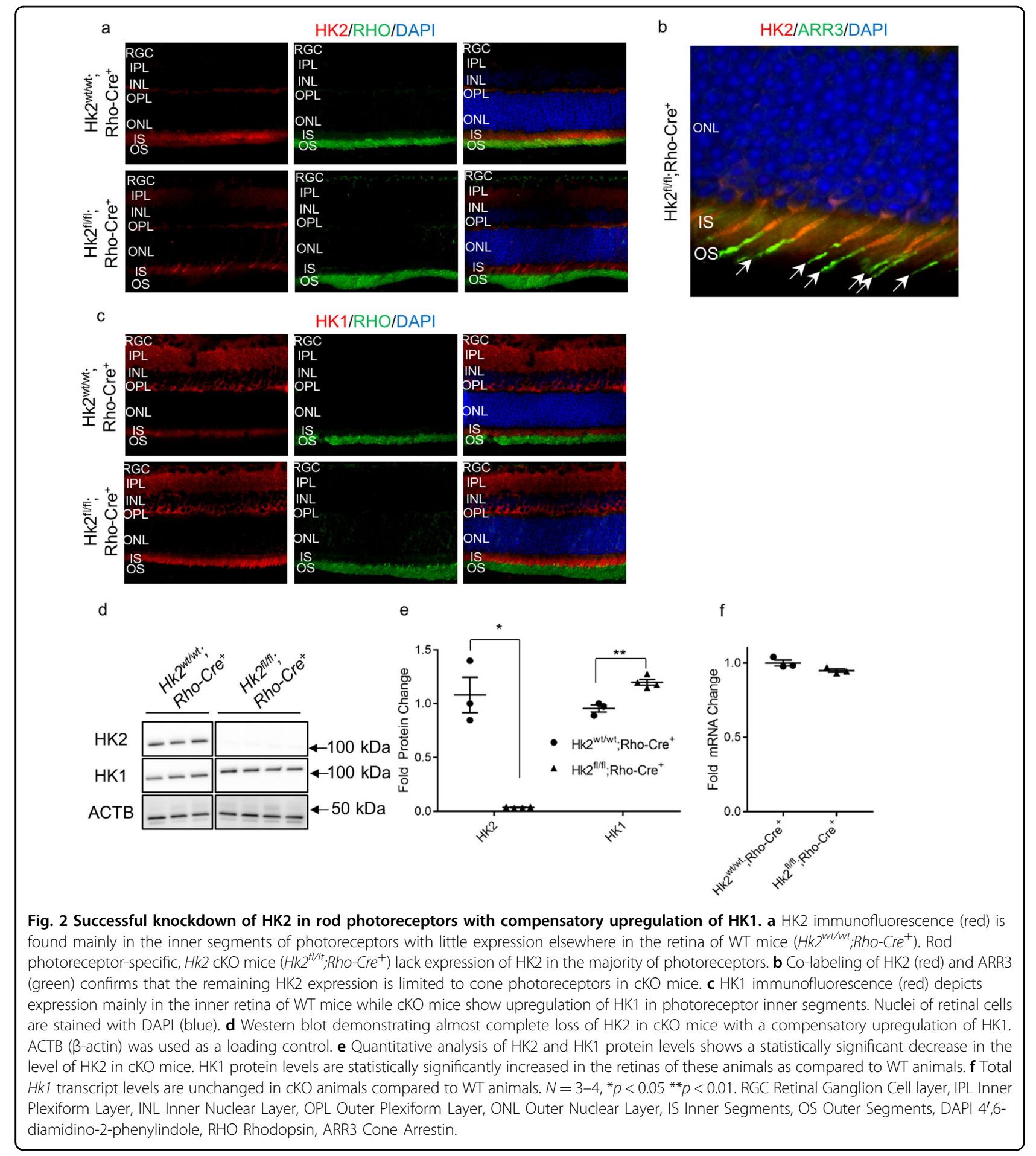

into lactate; therefore, a decrease in the total steady-state lactate levels would suggest significant perturbations to aerobic glycolysis ${ }^{37}$. No significant differences in total retinal lactate levels between $\mathrm{cKO}$ and WT animals were observed at 2 months of age (Fig. 5b). The concentration of ATP from total retinal lysates was assayed. We found a significant increase in total ATP levels in cKO animals compared to WT animals (Fig. 5c). PKM2, LDHA, and GLUT1 were assayed via Western blotting. No significant differences in the expression of any of these proteins was detected (Fig. 5d-i, Fig. S1). Collectively, these data demonstrate the lack of any major retinal metabolic reprogramming of glucose metabolism when $H k 2$ is genetically deleted in rod photoreceptors. 

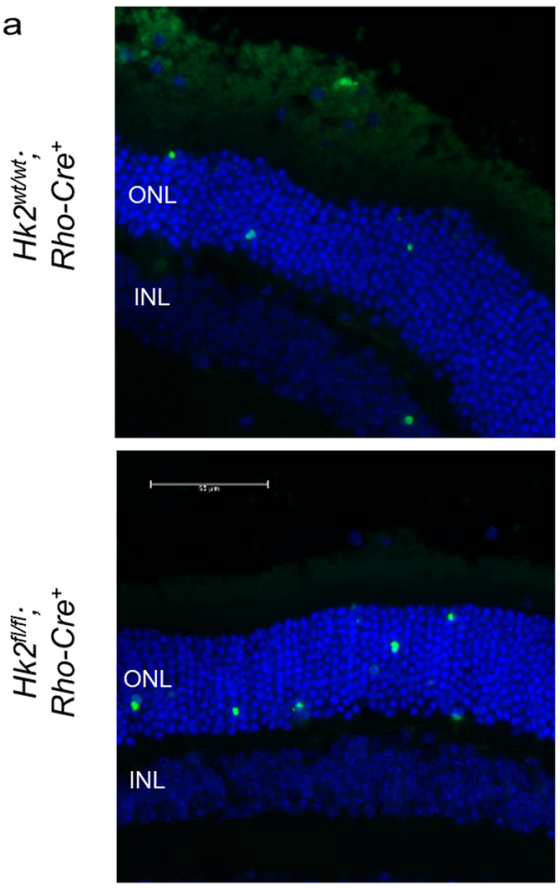

C

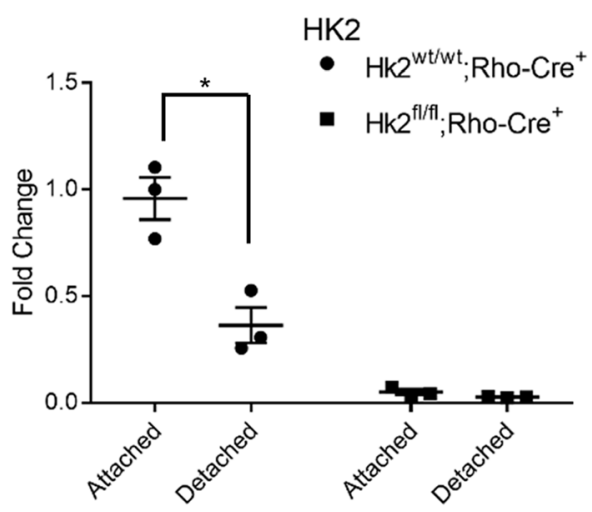

e

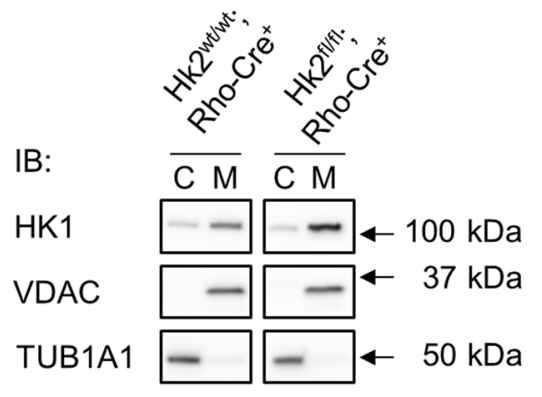

b

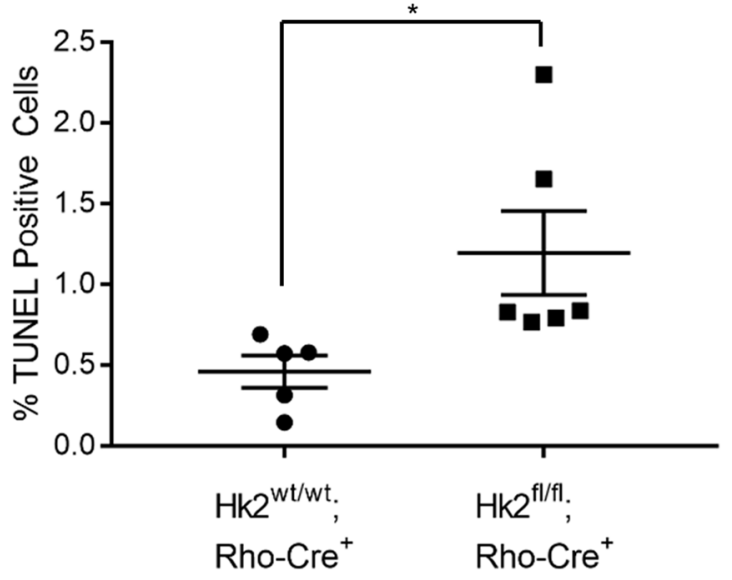

d

HK1
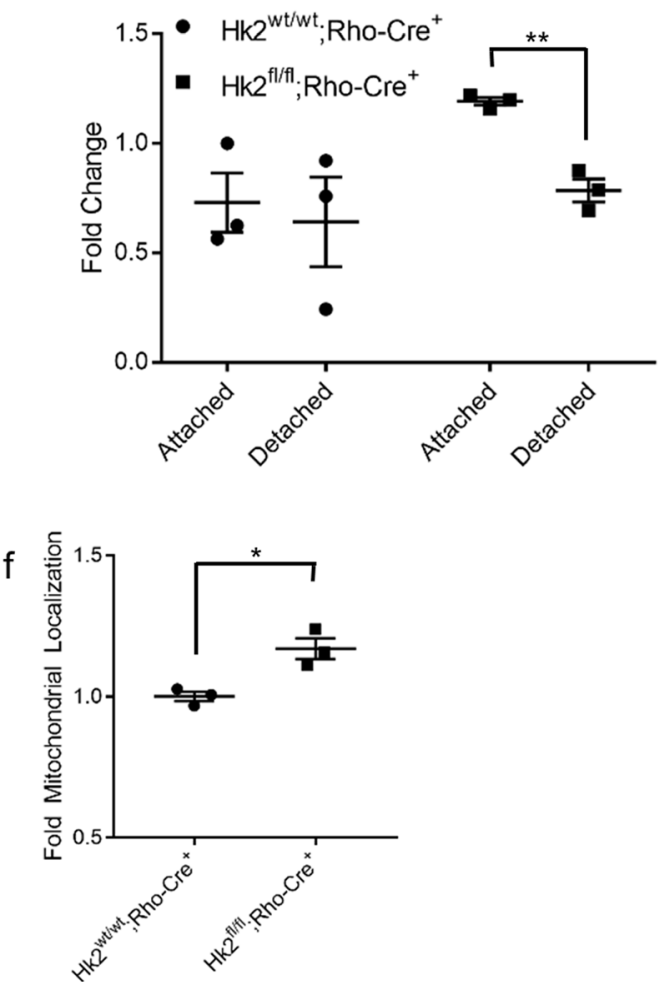

Fig. 3 HK2 regulates photoreceptor survival after retinal detachment. a Representative images of TUNEL-stained photoreceptors (green) in detached regions of mouse retina 3 days post-retinal detachment. Nuclei of cells within ONL (outer nuclear layer) and INL (inner nuclear layer) are stained with DAPI (blue). b Quantification of TUNEL-positive cells in the ONL. c Quantitative analysis of HK2 protein levels 3 days after retinal detachment. HK2 is down-regulated following retinal detachment in WT mice. d Quantitative analysis of HK1 protein levels 3 days after retinal detachment. HK1 is downregulated following retinal detachment in CKO mice only. e Representative Western blot for data quantified in f. f Total HK1 protein levels are significantly enriched in the mitochondrial fraction in CKO 3-days post-retinal detachment in cKO animals versus WT animals. $N=3-6$ animals per group, ${ }^{*} p<0.05,{ }^{* *} p<0.001$. 


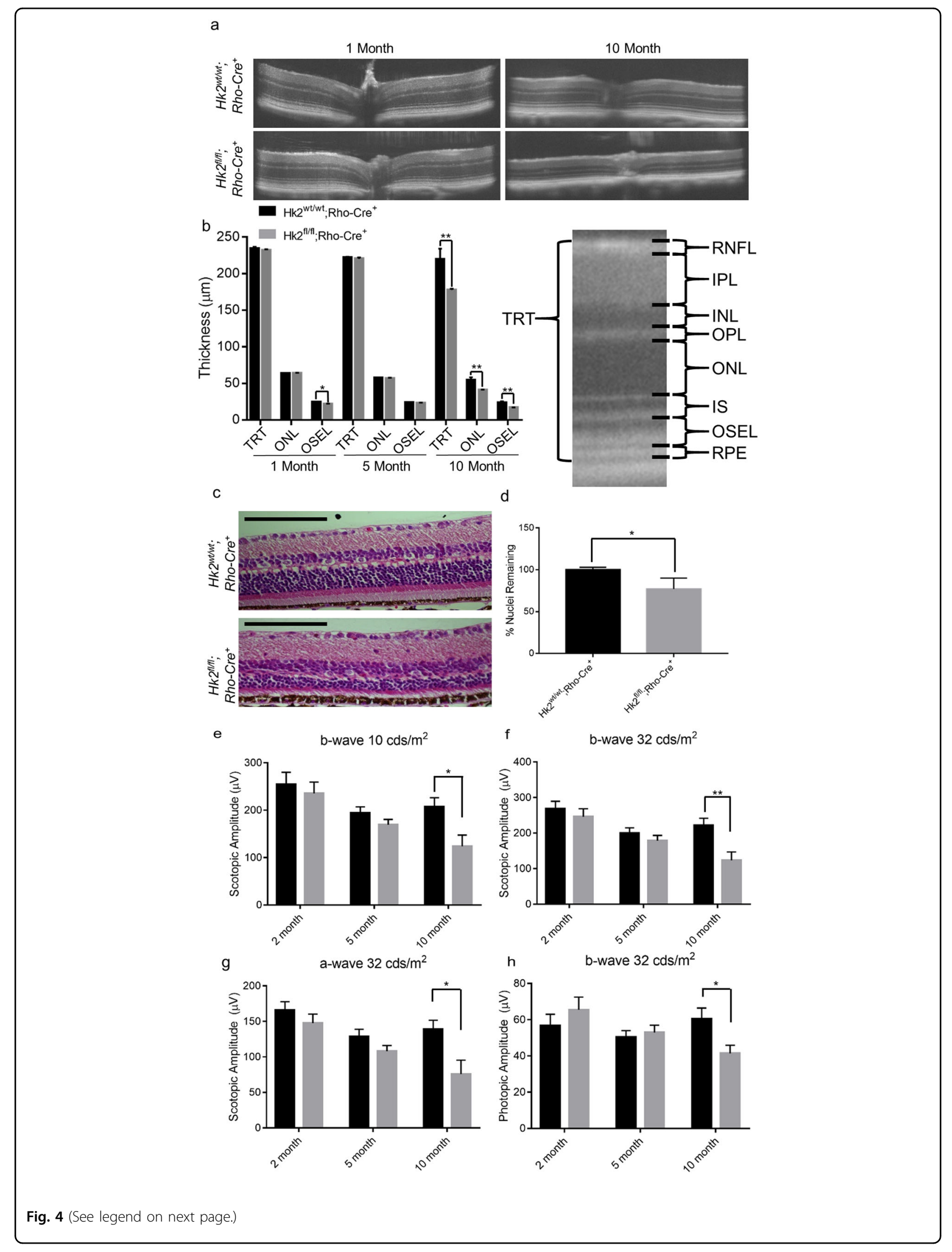


(see figure on previous page)

Fig. 4 HK2 is required for long-term survival of photoreceptors. a Representative OCT images of 1- and 10-month-old cKO mice. b Quantitative analysis of retinal layers from OCT images. TRT, ONL and OSEL are significantly thinner in CKO mice as compared to WT animals. Schematic of retinal layers measured using OCT. OSEL is the region between the inner segment boundary and the apical surface of the RPE. c Representative hematoxylin and eosin stained sections of 10-month-old cKO and WT mice. $\mathbf{d}$ ONL nuclei counts normalized to total inner retinal area as a percent of the WT mice. e-h ERG amplitudes at indicated illuminance. e- $\mathbf{g}$ Scotopic and $\mathbf{h}$ photopic measurements. Amplitude is significantly decreased in cKO animals at 10 months of age. $N=8-16,{ }^{*} p<0.05,{ }^{* *} p<0.01$. TRT total retinal thickness, RNFL retinal nerve fiber layer, IPL inner plexiform layer, INL inner nuclear layer, OPL outer plexiform layer, ONL Outer Nuclear Layer, IS inner segments, OSEL outer segment equivalent length, RPE retinal pigment epithelium.

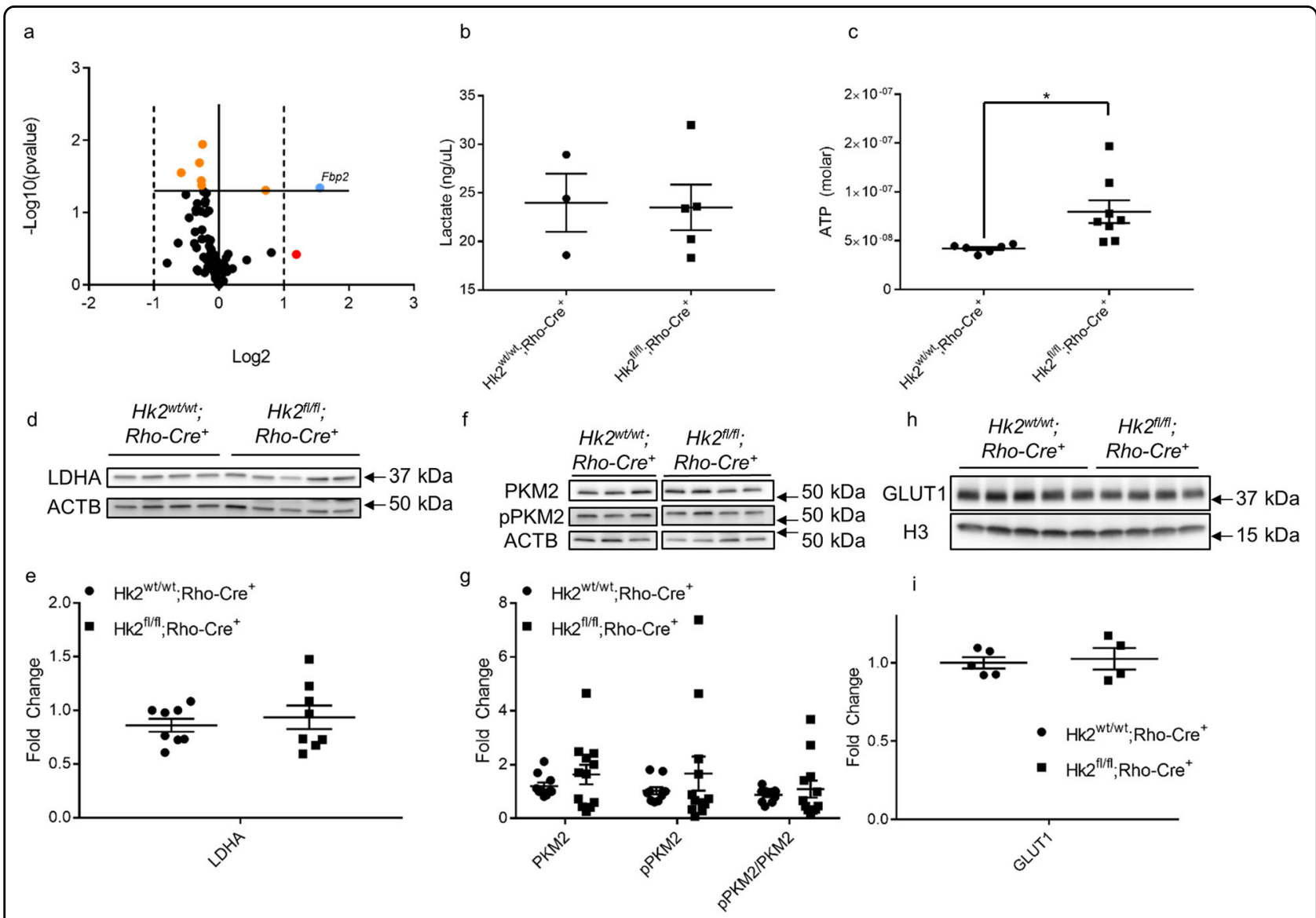

Fig. 5 Glucose metabolism is largely unchanged in rod photoreceptor-specific, Hk2 conditional knockout mice. a Volcano plot of 84 glucose metabolism gene expression as measured by qRT-PCR. The solid horizontal line indicates a $p$-value of 0.05 . The vertical dotted lines indicate a fold change of \pm 2 . Orange dots are significantly different. The red dot has an insignificant fold change greater than 2 . The blue dot shows a gene changed more than \pm 2 -fold and with a $p$-value of $<0.05$. Only Fbp2 is significantly changed $>2$-fold. $\mathbf{b}$ Total retinal lactate is unchanged between $c K O$ and WT mice measured at 6-8 weeks of age. c Total ATP content of the retina is significantly increased in cKO animals at 12 weeks of age. d, e Representative western blot and respective quantitative analysis confirms LDHA protein levels are unchanged after deletion of HK2 from rod photoreceptors. $\mathbf{f}, \mathbf{g}$ Representative Western blot of total PKM2 and p-PKM2 (Y105) and respective quantitative analyses demonstrates that these levels are unchanged in CKO animals as compared to WT mice. $\mathbf{h}$, i Representative Western blot and respective quantitative analysis shows no change in GLUT1 levels in CKO animals. $N=3-9$, mean + SEM.

\section{Discussion}

In this study, we show that HK2 translocates to mitochondria following retinal detachment and at the same time is significantly downregulated. This change in subcellular localization following nutrient deprivation stress in the retina may be mediated by activated retinal AKT.
We also show that inhibiting AKT phosphorylation using a broad PI3K inhibitor (LY294002) results in accumulation of HK2 in the cytosol. Treatment with LY294002 activates caspase 3,7 , and 8 , and reduces cell viability, possibly due to reduced HK2 fraction in the mitochondria. Rod-specific $H k 2$ deletion results in upregulation of 
Hk1 expression in photoreceptor inner segments. Under acute outer retinal stress induced by experimental retinal detachment, HK2-to-HK1 isoform switching increases rod photoreceptor susceptibility to cell death. This HK isoform switch also leads to degenerative changes in the outer retina with aging, as shown by the decrease in both ERG amplitudes and concurrent decline in retinal thickness measurements. Interestingly, only a single central metabolic gene, $F b p 2$, exhibited a significant change in expression. There were no significant changes in aerobic glycolysis observed in the retinas of $H k 2 \mathrm{cKO}$ mice except for an increase in total ATP content of the retina. These data indicate that increased HK1 expression in rods functionally replaces HK2 at the metabolic level, and since there were no overt changes to metabolism in photoreceptors it is likely that ATP catabolism may be altered in cKO animals. Under the experimental conditions used in these studies, the neuroprotective effect of HK2 during acute outer retinal stress and aging may be due to its ability to control ATP production and via its nonenzymatic activities ${ }^{17,18,20}$.

Deletion of HK2 from rod-photoreceptors led to an increase in total HK1 levels in the retina, specifically rod inner segments. Interestingly, $H k 1$ transcript levels are unchanged, suggesting the increase in total HK1 protein level is due to either increased protein stability, as photoreceptors appear to express low levels of HK1 at baseline, or to increased translation of existing $H k 1$ mRNA. Despite HK2 expression being associated with increased aerobic glycolysis, we were unable to detect any significant perturbations to aerobic glycolysis in retinas from HK2 cKO animals ${ }^{38,39}$. Lactate is almost exclusively produced by photoreceptors in the retina through aerobic glycolysis, however no significant changes in steady-state lactate production were observed in cKO retinas ${ }^{37}$. Furthermore, very few changes were observed in the expression of genes involved in central glucose metabolism. There was, however, a significant increase in total ATP content of the retina. This could mean that photoreceptors are producing more ATP, but not using this excess energy and wasting available nutrients. An alternate explanation may involve the difference in ATP utilization by HK2 and HK1. HK2 has two catalytic domains while HK1 only has a single catalytic domain. The $\mathrm{N}$-terminal domains of HK1 and HK2 are very similar, however the C-terminal domain of HK2 has a $K_{i}$ approximately 30 times greater, suggesting that HK2 is inhibited at much higher concentrations of glucose-6-phosophate ${ }^{40}$. This difference may mean that the increased ATP concentration observed in cKO retinas is due to less glucose being phosphorylated and therefore more ATP available. As we did not observe any significant changes in metabolism, an overall defect in ATP catabolism may explain this observation. Taken together, these data suggest that HK1 can nearly metabolically replace HK2. Future studies will focus on determining the steady-state levels of metabolites during glucose metabolism as well as flux of ${ }^{13} \mathrm{C}$-glucose as it is broken down through glycolysis and branching pathways to reveal the exact metabolic effects of HK2-to-HK1 substitution in photoreceptors.

As glucose is the primary fuel source for photoreceptors, an isoform of hexokinase is likely required to initiate the glycolytic cascade. All isoforms of hexokinase perform the same enzymatic reaction, phosphorylating glucose to glucose-6-phosphate. Yet, each isoform has differing enzymatic rates to account for varying responsibilities depending on cell type ${ }^{40}$. Interestingly, HK1 and HK2 have similar enzymatic constants and even though HK2 is believed to be critical for aerobic glycolysis, this enzymatic similarity may allow HK1 to maintain metabolic homeostasis and ultimately, to prevent significant transcriptional response in the absence of HK2. We could not detect any changes in LDHA, PKM2, or GLUT1 protein expression and when examining transcription of genes encoding for enzymes in central glucose metabolism, the only gene that was significantly altered in $H k 2$ cKO retinas was $F b p 2$. Enhanced fructose bisphosphatase (FBPase) activity and decreased fructose 2,6-bisphosphate (F2,6P) levels secondary to increased $F b p 2$ expression would be expected to decrease glycolytic flux and may maintain photoreceptor glucose homeostasis in $H k 2 \mathrm{cKO}$ retinas via opposing PFK1 activity, the critical enzyme for committing glucose-derived metabolites to glycolytic flux $^{41,42}$.

The data presented here suggest that the non-enzymatic functions of HK2 may be more important for preserving photoreceptors during acute stress than its enzymatic roles. Both HK1 and HK2 are maximally enzymatically active and preferentially use mitochondrial produced ATP to phosphorylate glucose ${ }^{43}$. Since a significant fraction of all HK2 is found in the cytosolic fraction, HK2 subcellular localization may be regulated to fine tune the amount of glucose entering glycolysis. At the same time, HK2 may also be performing a critical, non-enzymatic function in the cytosol unrelated to glycolysis. Further studies are needed to investigate the potentially distinct roles of HK2 in the cytosol, which may explain why HK2 is preferentially expressed in photoreceptors in the retina. One of the major non-enzymatic roles of HK2 is to inhibit binding of pro-apoptotic factors and prevent opening of the mPTP, which may explain why HK2 translocates to the mitochondrial fraction following retinal detachment. Interestingly, we observed a similar increase in HK1 enrichment in the mitochondrial fraction following retinal detachment in cKO animals. HK1 has some ability to stymie pro-apoptotic signaling, however, our data indicate that this function is not enough to prevent photoreceptor death in the same manner as $\mathrm{HK}^{44-46}$. In addition, HK2, 
but not HK1, has a consensus AKT phosphorylation site that when modified enhances binding to VDAC. Since HK1 lacks this ability it may also not be able to as robustly counter pro-apoptotic signaling as HK2. In our in vitro model, when HK2 translocation is partially blocked using the PI3K inhibitor LY294002, $661 \mathrm{~W}$ cells activate caspases and undergo cell death. While inhibiting the PI3K signaling cascade alters numerous intracellular pathways that may be important in cell survival, our data suggests that HK2 binding to mitochondria is at least partially responsible for inhibiting apoptosis, similar to what was shown in other experimental models ${ }^{21}$. Experimental retinal detachment induces acute apoptotic stress on photoreceptors, which is initially mediated by the Fas signaling cascade ${ }^{31,35}$. Previous work has shown that in addition to extrinsic pathway, Fas signaling acts through the intrinsic, mitochondrial mediated apoptosis pathway when XIAP is expressed ${ }^{47}$. Photoreceptors express XIAP early during retinal maturation which increases in mature retina $^{48,49}$. XIAP gene therapy increases photoreceptor survival after retinal detachment ${ }^{50}$. In addition, retinal detachment has been shown to activate both extrinsic and intrinsic death signaling pathways in the retina ${ }^{31,35}$. Activation of the intrinsic pathway was observed after experimental retinal detachment via increased caspase 9 activity, which could be blocked either by a small peptide inhibitor of the FAS receptor or through injection of neutralizing antibodies to either FasL or the FAS receptor $^{31,35}$. Further work to determine the exact mechanism by which HK2 promotes cell survival is necessary to understand the relationship between HK2 translocation to the mitochondria and apoptotic progression.

Photoreceptors lacking HK2 are more susceptible to acute nutrient deprivation caused by retinal detachment. Since HK1 replaces HK2 in cKO photoreceptors and has a similar affinity for glucose when compared to HK2, this susceptibility to nutrient deprivation is likely not related to metabolizing the limited amount of glucose in the detached retina ${ }^{43}$. Interestingly, HK2 protein levels drop precipitously 3 days after retinal detachment in WT animals, whereas a decrease in $H k 2$ transcript expression can be detected as early as 1-day post detachment. Likewise, when HK2 is replaced by HK1 in Hk2 cKO retinas, a robust decrease of $\mathrm{HK} 1$ expression was also observed after retinal detachment, in contrast to stable HK1 levels in WT retinas. These findings indicate that HK levels are regulated in rod photoreceptors during stress at both the protein and gene expression levels. The exact mechanisms of how HK2 production or degradation is regulated need to be explored further to fully understand its role for neuroprotection. Regardless of what is precipitating HK2 loss, it may be an attempt to catabolize intracellular proteins through autophagy to produce additional energy substrates and prolong survival in a nutrient deficient state. The degradation of HK2 during metabolic stress may disturb the intricate balance of apoptotic inhibition and energy production, uncoupling metabolic stress from cell death signaling and increasing the sensitivity of photoreceptors to nutrient deprivation. Raising the overall levels or preventing the degradation of HK2 may be beneficial for boosting photoreceptor survival during nutrient deprivation.

Even though HK1 appears to functionally replace HK2 at the metabolic level, $H k 2 \mathrm{cKO}$ retinas still developed an age-related phenotype. Similar to a previous report, we observed rod dysfunction in aged $H k 2 \mathrm{cKO}$ mice as measured by $\mathrm{ERG}^{28}$. The functional phenotype identified by Petit and colleagues was not associated with any morphological change in retinal structure. In contrast, we show that rod ERG dysfunction was associated with significant outer retinal abnormalities anatomically. This difference may be due to variations in genetic background between the two $H k 2$ cKO strains. We screened our animals for the $r d 8$ mutation to rule out the possibility of background degeneration and found that our $H k 2 \mathrm{cKO}$ animals lack the $r d 8$ allele. In addition, the phenotype of the $r d 8$ mutation has a very rapid onset, around 6 weeks of age, and is characterized by focal retinal folds, pseudorosettes, and retinal degeneration ${ }^{51}$. The phenotype observed in the HK2 cKO mice here was a slowly progressing degeneration and is not characteristic of the $r d 8$ mutation or other common background degenerative retinal diseases in mice ${ }^{51}$.

Our results suggest that HK2 has an essential function in preserving photoreceptors during aging. The time points we examined demonstrate that the onset of retinal degeneration in HK2 cKO animals is delayed. Our functional analysis by ERG and in vivo anatomic analysis by OCT at 5 months of age show that the overall retinal health is normal at this time. Thus, any potential degeneration was below the threshold of detection or had not yet begun. By 10 months of age there was a dramatic decrease in ONL and OSEL thickness. Interestingly, others have shown that the transcriptional profile of rod photoreceptors begins to change by 5 months of age ${ }^{52}$. Parapuram and colleagues found differential regulation of genes belonging to many different signaling cascades, including apoptosis, as animals age. At the same time, photoreceptors begin to accumulate significant oxidative damage with age, which may become toxic and increase apoptotic signaling ${ }^{53}$. The combination of loss of HK2, changes in gene regulation, and accumulation of oxidative injury may result in an imbalance of apoptotic signaling leading to delayed photoreceptor degeneration in our mouse model. Further studies are needed to determine the time course of photoreceptor degeneration in the $H k 2 \mathrm{cKO}$ animals with age and investigate the enzymatic and non-enzymatic functions of HK2 in aging photoreceptor cells. 


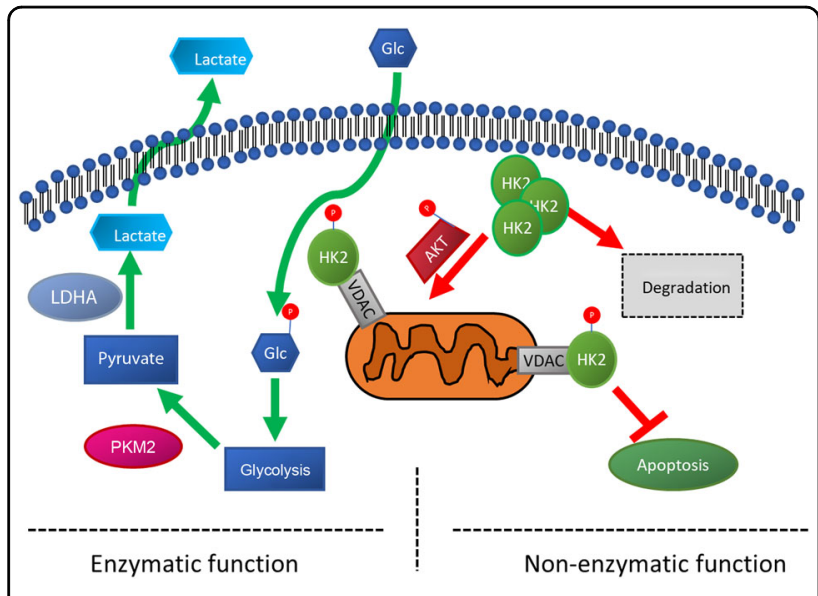

Fig. 6 HK2 regulates aerobic glycolysis in photoreceptors via its enzymatic activity under normal physiologic conditions. Glucose deprivation leads to redistribution of HK2 from cytosol to mitochondria and AKT-mediated VDAC binding. This non-ezymatic function of HK2 is critical for preventing photoreceptor apoptosis during nutrient stress.

Based on our data, we propose the following model for HK2 function in photoreceptors (Fig. 6). Following retinal detachment, glucose levels drop precipitously. This results in a redistribution of $\mathrm{HK} 2$ to the mitochondrial fraction. Concurrently, AKT is activated, phosphorylating HK2 to enhance binding to VDAC. This serves to prevent the binding of pro-apoptotic factors to the mitochondria and prevent apoptotic signaling. As total HK2 drops significantly after retinal detachment, the neuroprotective effect of HK2 mediated by mitochondrial regulation of apoptosis may be limited. Determining the interactions between AKT/VDAC/HK2 in the retina will be important for verifying this model. These experiments will enhance our understanding of the role of HK2 in both anti-apoptotic and pro-survival functions in photoreceptors.

We previously showed that reprogramming photoreceptor metabolism via modulating a key regulatory enzyme of aerobic glycolysis, PKM2, is a novel therapeutic strategy. Interestingly, this effect seems to be due mostly to the primary function of PKM1/2, its enzymatic activity with respect to glycolysis. Data presented in this study demonstrate that modulating the other critical regulator of aerobic glycolysis, HK2, may also increase photoreceptor survival during metabolic stress. Compared to our work regarding PKM2, the role of HK2 on photoreceptor survival appears to be linked to its secondary, non-enzymatic roles surrounding apoptosis instead of through its primary role in glycolysis. It will be useful to further examine any potential links between HK2 and PKM2 in future studies, specifically if and how they interact during their roles in glycolysis. Determining the exact role of HK2 during nutrient stress has the potential to reveal novel molecular pathways that can be targeted for photoreceptor neuroprotection.

\section{Methods \\ Animals}

All animals were treated in accordance with the Association for Research in Vision and Ophthalmology (ARVO) Statement for the Use of Animals in Ophthalmic and Vision Research. The protocol was approved by the University Committee on Use and Care of Animals of the University of Michigan (Protocol number: PRO00007463). All animals were maintained at room temperature in a 12/ 12-h light/dark cycle. Mice harboring a floxed $H k 2$ gene were a gift from Dr. Mohanish Deshmukh and Dr. Timothy Gershon $^{27}$. These mice were crossed to mice carrying a Cre-recombinase gene under the control of the rhodopsin promoter $^{26}$. The mice were maintained on a C57BL/6 background and were confirmed to not carry the $r d 8$ mutation. $\mathrm{Hk}^{w t / w t}$;Rho-Cre (Wildtype, WT) and $H k 2^{f l l f l}$; $\mathrm{Rho}^{-\mathrm{Cre}}{ }^{+}$(conditional knockout, cKO) mice were generated.

\section{Sub-cellular fractionation}

Rodent retinas were isolated from euthanized animals using the "Cut and Pick" (or Winkling) method while being careful to avoid collecting $\mathrm{RPE}^{54}$. The retinas were fractionated into cytosolic and post-cytosolic fractions using the Subcellular Protein Fractionation Kit for Tissues (Thermo Fisher Scientific; Waltham, MA, USA; Cat\# 87790) following a modified manufacturer's protocol. Two retinas were pooled for each sample with a total of three or four samples for each timepoint or condition and homogenized in cytoplasmic extraction buffer (CEB) supplemented with protease ( Halt $^{\mathrm{TM}}$ Protease Inhibitor Cocktail, Thermo Fisher Scientific, Cat\# 87786) and phosphatase (Halt ${ }^{\mathrm{TM}}$ Phosphatase Inhibitor Cocktail, Thermo Fisher Scientific, Cat\# 78420) inhibitors using 30-40 strokes with the "B" pestle of a Dounce homogenizer. Lysate was then centrifuged at $4{ }^{\circ} \mathrm{C}$ for $10 \mathrm{~min}$ at 10,000 relative centrifugal force (RCF). The supernatant (cytosolic fraction) was saved and the pellet was sonicated at $20 \%$ amplitude with $1 \mathrm{~s}$ on/off pulse for $10 \mathrm{~s}$ in RIPA lysis buffer (Thermo Fisher Scientific, Cat\# 89900) supplemented with protease and phosphatase inhibitors. This lysate was then centrifuged at $4{ }^{\circ} \mathrm{C}$ for $10 \mathrm{~min}$ at 10,000RCF. The supernatant (mitochondrial enriched fraction) was saved and the pellet was discarded. Equivalent microgram of protein from each fraction was loaded and run on Western blot to determine relative HK2 or HK1 levels between fractions.

The $661 \mathrm{~W}$ photoreceptor cell line was a generous gift from Dr. Muayyad Al-Ubaidi ${ }^{55}$. These cells have not been recently tested for authenticity or mycoplasma contamination. In all, $2 \times 10^{6} 661 \mathrm{~W}$ cells were seeded $24 \mathrm{~h}$ 
prior to treatment. Cells were fractionated using the Subcellular Protein Fractionation Kit for Cultured Cells (Thermo Fisher Scientific, Cat\# 78840) as above except cells were trypsinized, pelleted, rinsed with $1 \mathrm{X}$ phosphate buffered saline (PBS), re-pelleted before being resuspended in CEB for a 10 -min incubation at $4{ }^{\circ} \mathrm{C}$. The remaining steps were identical. Cells were cultured as described previously and treated with DMSO or LY294002 (Cell Signaling Technology; Denver, MA, USA, Cat\# 9901, $50 \mu \mathrm{M}, 5 \mathrm{~mL}$ ) in glucose-free DMEM (Thermo Fisher Scientific; Cat \# 11966025) supplemented with $5.5 \mathrm{mM}$ Glucose for $1.5 \mathrm{~h}$ prior to fractionation ${ }^{56}$. Wholecell lysates were also collected to verify loss of p-AKT after $1.5 \mathrm{~h}$ of LY294002 treatment. Equivalent microgram of protein from each fraction was loaded and run on Western blot to determine relative HK2 levels between fractions.

\section{Caspase activation and cell viability assays}

In all, $661 \mathrm{~W}$ cells were seeded at a density of 10,000 cells/well in each well of a 96-well plate then treated $24 \mathrm{~h}$ later with DMSO, $50 \mu \mathrm{M}$ LY294002, or media alone for $6 \mathrm{~h}$. Caspase activity was assayed using the Caspase-Glo® 3/7 or 8 Assay System (Promega; Madison, WI, USA, Cat\# G8090 or G8200) and cell viability was measured using the CellTiter-Glo ${ }^{\circledR}$ Luminescent Cell Viability Assay (Promega, Cat\# G7570) following the manufacturer's supplied instructions. Briefly, cells were treated for the desired length of time and then prepared reagent was added directly to each assay well, including blank wells containing media alone or media containing either DMSO or LY294002. The reaction was allowed to incubate for $15 \mathrm{~min}$ before assaying using a luminometer.

\section{Western blotting}

After harvest, retinas were homogenized in RIPA lysis buffer supplemented with protease and phosphatase inhibitors. Homogenization was performed using a sonicator set at $20 \%$ amplitude with $1 \mathrm{~s}$ on/off pulse for $10 \mathrm{~s}$ followed by centrifugation at $10,000 \mathrm{RCF}$ at $4{ }^{\circ} \mathrm{C}$ for $10 \mathrm{~min}$. Clarified lysates had protein content estimated using the Pierce ${ }^{\mathrm{TM}}$ BCA Protein Assay Kit (Thermo Fisher Scientific, Cat\# 23225). In all, $15 \mu$ g of total protein was diluted in Laemmli buffer (Bio-Rad; Hercules, CA USA, Cat\# 1610747) supplemented with $\beta$-mercaptoethanol (Millipore-Sigma; St. Louis, MO USA, Cat\# M6250). Denatured protein samples were run on $4-20 \%$ or $10 \%$ Mini-PROTEAN ${ }^{\circledR}$ TGX $^{\mathrm{TM}}$ Precast Protein Gel (Bio-Rad, Cat\# 4561094). Protein was transferred using either a wettransfer $\left(100 \mathrm{~V}\right.$ for $1 \mathrm{~h}$ at $\left.4{ }^{\circ} \mathrm{C}\right)$ or the Trans-Blot ${ }^{\circ}$ Turbo $^{\mathrm{TM}}$ Transfer System $(25 \mathrm{~V}$ for $30 \mathrm{~min})$ (Bio-Rad, Cat\# 1704150). After transfer, the PVDF membranes were blocked using $5 \%$ non-fat dry milk in TBS-T (Tris-buffered Saline (Bio-Rad, Cat\# 1706435) supplemented with
Table 1 Antibodies used.

\begin{tabular}{|c|c|c|}
\hline Protein target & Dilution & Manufacturer \\
\hline HK1 & 1:1000 (WB), 1:2000 (IF) & CST \#2024 \\
\hline HK2 & $1: 1000(\mathrm{WB}, \mathrm{IF})$ & CST \#2867 \\
\hline ARR3 & $1: 200$ (IF) & SCB \#SC-293296 \\
\hline LDHA & 1:1000 (WB) & CST \#2012 \\
\hline PKM2 & 1:1000 (WB) & CST \#4053 \\
\hline GLUT1 & 1:1000 (WB), 1:100 (IF) & Abcam \#ab15309 \\
\hline p-PKM2 (Y105) & 1:1000 (WB) & CST \#3827 \\
\hline VDAC & 1:1000 (WB) & CST \#4866 \\
\hline AKT & 1:1000 (WB) & CST \#4691 \\
\hline p-AKT (S473) & 1:1000 (WB) & CST \#9271 \\
\hline $\mathrm{RHO}$ & 1:1000 (IF) & Abcam \#ab5417 \\
\hline ACTB & 1:10,000 (WB) & MilliporeSigma \#A5316 \\
\hline TUB1A1 & $1: 2000$ (WB) & MillporeSigma \#T6199 \\
\hline HRP-anti-rabbit & 1:2000 (WB) & Dako \#P0447 \\
\hline HRP-anti-mouse & $1: 2000$ (WB) & Dako \#P0448 \\
\hline Alexa 594 Anti-rabbit & 1:1000 (IF) & ThermoFisher \#A11037 \\
\hline Alexa 488 Anti-mouse & $1: 1000$ (IF) & ThermoFisher \#A10680 \\
\hline
\end{tabular}

CST cell signaling technologies, SCT Santa Cruz Biotechnology, WB western blot, IF Immunofluorescence, HK1 Hexokinase 1, HK2 Hexokinase 2, ARR3 Cone Arrestin, LDHA lactate dehydrogenase A, RHO Rhodopsin, ACTB $\beta$-Actin, HRP Horseradish Peroxidase, PKM2 Pyruvate kinase muscle isozyme 2, AKT Protein kinase B, TUB1A1 a-tubulin, VDAC voltage dependent anion channel, GLUT1 glucose transporter 1 .

0.1\% Tween-20 (Thermo Fisher Scientific, Cat\# 28320)). Primary antibodies were diluted according to Table 1 in 5\% Bovine Serum Albumin (BSA; (Millipore-Sigma, Cat\# A9647)) in TBS-T and blocked membranes were incubated overnight at $4{ }^{\circ} \mathrm{C}$ with gentle agitation. Membranes were then washed and incubated with appropriate secondary antibody diluted in 5\% BSA in TBST for $1 \mathrm{~h}$ at room temperature. Protein bands were detected using the SuperSignal $^{\mathrm{TM}}$ West Dura/Femto Extended Duration Substrate (Thermo Fisher Scientific, Cat\# 34075 and 34094) with an Azure c500 imaging system (Azure Biosystems; Dublin, CA USA). Densitometry was performed using the Azure Spot program (Azure Biosystems; Dublin, CA USA).

\section{Immunofluorescence}

Mouse eyes were enucleated and fixed in $4 \%$ formaldehyde (Polysciences; Warrington, PA, USA, Cat\# 18814 ) overnight at $4{ }^{\circ} \mathrm{C}$. Fixed eyes were embedded in paraffin and sectioned $6 \mu \mathrm{m}$ thick. Sections were deparaffinized and antigen retrieval was performed following standard procedures. Blocking was performed with $1 \%$ BSA in PBST supplemented with 10\% Normal Goat 
Serum (NGS) for $1 \mathrm{~h}$ before adding primary antibody (Table 1) diluted in 1\% BSA supplemented with 1\% NGS overnight at $4{ }^{\circ} \mathrm{C}$. Slides were then washed in PBS and then with $1 \%$ BSA in PBST supplemented with 1\% NGS before appropriate secondary antibody was added (Table 1) and slides were incubated at room temperature for $1 \mathrm{~h}$. Finally, slides were washed with PBS and counterstained with ProLong ${ }^{\mathrm{TM}}$ Gold Antifade Mountant with DAPI (Thermo Fisher Scientific, Cat\# P36930). Slides were imaged using a Leica DM6000 microscope with a 40X objective.

\section{qRT-PCR}

Four Retinas for each group (WT or cKO) were extracted as described above and immediately immersed in RNAlater (Qiagen; Hilden, Germany, Cat\# 76104). Total RNA was extracted using the RNeasy Mini Kit (Qiagen, Cat\# 74104) following the manufacturer's instructions. RNA quantity and quality were assessed with a Nanodrop 1000 (Thermo Fisher Scientific). In all, 400 ng of total RNA was reverse transcribed using the $\mathrm{RT}^{2}$ First Strand Kit (Qiagen, Cat\# 330401). Transcriptional changes in metabolic pathways was assessed using the $\mathrm{RT}^{2}$ Profiler PCR Array for Mouse Glucose Metabolism (Qiagen, Cat\# 330231 PAMM-006ZA) following the manufacturer's instructions. In all, $102 \mu \mathrm{L}$ of cDNA was pre-mixed with $650 \mu \mathrm{L}$ of $2 \mathrm{X}$ SYBR Green master mix (Qiagen, Cat\# 330503) and $548 \mu \mathrm{L}$ of ddH2O. In all, $10 \mu \mathrm{L}$ of each pre-mix was added to each well of the 384 well plate following the manufacturer's instructions. The plates were thermocycled using a CFX384 thermocycler (Bio-Rad) following the provided cycling parameters. The geometric mean of the Ct values for Actb, Gapdh, and Hsp90ab1 was used for relative quantitation using the $2^{-\Delta \Delta \mathrm{Ct}}$ method. Total mouse $H k 1$ transcript levels were measured using Qiagen Quantitect primer QT01039598. Total $H k 2$ transcript levels were measured using the following primer pair: 5'- CTCCATCCCACAGGAGGTT A- $3^{\prime}$ and $5^{\prime}-$ GCTTCCTTCAGCAAGGTGAC- $3^{\prime}$.

\section{Functional assessment}

Functional assessment of mouse vision was performed as described previously ${ }^{7}$. Total retinal function was measured using electroretinography (ERG). Mice were anesthetized and the ERG response was measured using the Diagnosys Celeris ERG system (Diagnosys LLC, Lowell, MA, USA). In vivo retinal thickness was assessed using the Envisu-R SD-OCT imager (Leica Microsystems Inc., Buffalo Grove, IL, USA). Average thickness of various retinal layers was assessed using Diver 1.0 (Leica Microsystems Inc). Four points $200 \mu \mathrm{m}$ from the optic nerve head were used to measure layer thickness and the results were averaged. Total retinal thickness (TRT), outer nuclear layer (ONL) thickness and outer segment equivalent length (OSEL) were determined. OSEL is defined as the length from the ends of the inner segment boundary to the surface of the $\mathrm{RPE}^{7,57}$.

\section{Retinal detachment, TUNEL staining, and ONL counts}

Experimental neurosensory retinal detachment was induced in mice as described previously ${ }^{7}$. Briefly, animals were anesthetized and a sclerotomy was performed using a 25G micro-vitrealretinal blade. A 35G beveled cannula (World Precision Instruments; Sarasota, FL, USA, Cat\# NF35BV-2), attached to a NanoFil-100 syringe (World Precision Instruments, Cat\# NANOFIL-100) was used to inject $2-3 \mu \mathrm{L}$ of Healon (1\% Hyaluronic Acid) (Abbott Medical Optics; Santa Ana, CA, USA, Cat\# 05047450842) between the photoreceptor and RPE layers. Care was taken to detach approximately half of the retina in each animal. Only the sclerotomy was performed on the fellow eye as a control. After 3 days, animals were sacrificed and whole eyes were extracted and embedded in paraffin for sectioning as described above. TUNEL staining was performed using the DeadEnd ${ }^{\mathrm{TM}}$ Fluorometric TUNEL System (Promega Cat\# G3250) and sections were counterstained using ProLong Gold Mountant with DAPI. Stained sections were imaged with a Leica DM6000 microscope using a 40X objective. TUNEL positive cells were manually counted across the detached portion of the retina. Counts were normalized to the total number of nuclei in the outer nuclear layer in the detached region, counted manually or with an automated cell counting macro using Image ${ }^{7,56}$.

\section{Total retinal lactate quantification}

Total Retinal Lactate was quantified using the Lactate Assay Kit (Millipore-Sigma, Cat \# MAK064) following the manufacturer's supplied protocol. Animals were euthanized and neural retina was extracted as described above and briefly washed in PBS to remove any adhered vitreous. The retinas from both eyes of each animal were pooled and homogenized using the provided Lactate Assay Buffer. A sample of homogenate was retained for total protein quantification to normalize samples before de-proteinization using the Amicon Ultra-0.5 Centrifugal Filter Unit (Millipore-Sigma, Cat\# UFC501024), which has a molecular weight cut-off of $10 \mathrm{kDa}$. In all, $10 \mu \mathrm{L}$ of sample was loaded in duplicate for both the colorimetric and fluorometric assay. A standard lactate concentration curve was plotted for each assay and the concentration of each sample was determined from this curve. The concentration of lactate $(\mathrm{ng} / \mu \mathrm{L})$ was normalized by the total sample protein concentration.

\section{Total ATP quantification}

Total ATP levels were determined from total retinal lysates using the ATP Bioluminescence Assay Kit CLS II (Millipore-Sigma, St. Louis, MO, Cat\# 11699695001) 
following the manufacturer's supplied protocol. Briefly, individual retinas were harvested and flash frozen. Each retina was homogenized in $90 \mu \mathrm{L}$ RIPA using sonication as described above. Samples were diluted 1:1 with assay buffer and luminescence was read and compared to a standard ATP concentration curve to determine ATP content. ATP levels were normalized to total protein content.

\section{Statistical analysis}

All experiments were replicated at least twice to confirm results. Both male and female animals were used. The size of cohorts is based on power calculations derived from our experience with the retinal detachment model. Our data strongly suggest that group sizes of 5 will allow us to detect at least a $30 \%$ difference between groups with power of $90 \%$ and $p<0.05$. No samples were excluded from analysis. Animals were not randomly assigned. Experimenters were single-masked to the identify of samples during analysis but not during collection. Statistical analysis was performed comparing WT to cKO samples using a two-tailed student's $t$-test with either Excel or Prism 7.0. Results with a $p$-value $\leq 0.05$ were considered significant. Data are displayed as mean \pm SEM, and the number of replicates is indicated in each figure legend.

\section{Acknowledgements}

We would like to acknowledge Sarah Sheskey for her help performing ERG. This work was supported by the Macula Society, E. Matilda Ziegler Foundation, and the NIH/NEI (5K08EY023982 and R01EY029675). This work utilized the Vision Research Core funded by P30 EY007003 from the National Eye Institute.

\section{Data availability}

All data will be provided upon reasonable request to the corresponding author.

\section{Conflict of Interest}

The authors declare that they have no conflict of interest.

\section{Publisher's note}

Springer Nature remains neutral with regard to jurisdictional claims in published maps and institutional affiliations.

Supplementary Information accompanies this paper at (https://doi.org/ 10.1038/s41419-020-2638-2).

Received: 30 September 2019 Revised: 15 May 2020 Accepted: 18 May 2020

Published online: 04 June 2020

\section{References}

1. Pardue, M. T. \& Allen, R. S. Neuroprotective strategies for retinal disease. Prog. Retin. Eye Res. 65, 50-76 (2018).

2. Boesze-Battaglia, K. \& Goldberg, A. F. Photoreceptor renewal: a role for peripherin/rds. Int. Rev. Cytol. 217, 183-225 (2002).

3. Hartong, D. T. et al. Insights from retinitis pigmentosa into the roles of isocitrate dehydrogenases in the Krebs cycle. Nat. Genet 40, 1230-1234 (2008).

4. Wang, F. et al. A missense mutation in HK1 leads to autosomal dominant retinitis pigmentosa. Invest Ophthalmol. Vis. Sci. 55, 7159-7164 (2014).
5. Bowne, S. J. et al. Mutations in the inosine monophosphate dehydrogenase 1 gene (IMPDH1) cause the RP10 form of autosomal dominant retinitis pigmentosa. Hum. Mol. Genet 11, 559-568 (2002).

6. Leveillard, T., Philp, N. J. \& Sennlaub, F. Is retinal metabolic dysfunction at the center of the pathogenesis of age-related macular degeneration? Int. J. Mol. Sci. 20, 762 (2019).

7. Wubben, T. J. et al. Photoreceptor metabolic reprogramming provides survival advantage in acute stress while causing chronic degeneration. Sci. Rep. 7, 17863 (2017).

8. Zhang, L. et al. Reprogramming metabolism by targeting sirtuin 6 attenuates retinal degeneration. J. Clin. Invest 126, 4659-4673 (2016).

9. Xu, L., Kong, L., Wang, J. \& Ash, J. D. Stimulation of AMPK prevents degeneration of photoreceptors and the retinal pigment epithelium. Proc. Natl Acad. Sci. USA 115, 10475-10480 (2018).

10. Wang, W. et al. Two-step reactivation of dormant cones in retinitis pigmentosa. Cell Rep. 15, 372-385 (2016).

11. Kooragayala, K. et al. Quantification of oxygen consumption in retina ex vivo demonstrates limited reserve capacity of photoreceptor mitochondria. Invest Ophthalmol. Vis. Sci. 56, 8428-8436 (2015).

12. Winkler, B. S. Glycolytic and oxidative metabolism in relation to retinal function. J. Gen. Physiol. 77, 667-692 (1981).

13. $\mathrm{Ng}, \mathrm{S}$. K. et al. Cancer-like metabolism of the mammalian retina. Clin. Exp. Ophthalmol. 43, 367-376 (2015).

14. Wang, L., Tornquist, P. \& Bill, A. Glucose metabolism in pig outer retina in light and darkness. Acta Physiol. Scand. 160, 75-81 (1997).

15. Casson, R. J., Chidlow, G., Han, G. \& Wood, J. P. An explanation for the Warburg effect in the adult mammalian retina. Clin. Exp. Ophthalmol. 41, 517 (2013).

16. Vander Heiden, M. G., Cantley, L. C. \& Thompson, C. B. Understanding the Warburg effect: the metabolic requirements of cell proliferation. Science $\mathbf{3 2 4}$, 1029-1033 (2009).

17. Gall, J. M. et al. Hexokinase regulates Bax-mediated mitochondrial membrane injury following ischemic stress. Kidney Int. 79, 1207-1216 (2011).

18. Majewski, N. et al. Hexokinase-mitochondria interaction mediated by Akt is required to inhibit apoptosis in the presence or absence of Bax and Bak. Mol. Cell 16, 819-830 (2004).

19. Pastorino, J. G., Shulga, N. \& Hoek, J. B. Mitochondrial binding of hexokinase II inhibits Bax-induced cytochrome c release and apoptosis. J. Biol. Chem. 277, 7610-7618 (2002).

20. Miyamoto, S., Murphy, A. N. \& Brown, J. H. Akt mediates mitochondrial protection in cardiomyocytes through phosphorylation of mitochondrial hexokinase-ll. Cell Death Differ. 15, 521-529 (2008).

21. Rajala, A., Gupta, V. K., Anderson, R. E. \& Rajala, R. V. Light activation of the insulin receptor regulates mitochondrial hexokinase. A possible mechanism of retinal neuroprotection. Mitochondrion 13, 566-576 (2013).

22. Al-Ubaidi, M. R., Matsumoto, H., Kurono, S. \& Singh, A. Proteomics profiling of the cone photoreceptor cell line, 661W. Adv. Exp. Med Biol. 613, 301-311 (2008).

23. Tan, E. et al. Expression of cone-photoreceptor-specific antigens in a cell line derived from retinal tumors in transgenic mice. Invest. Ophthalmol. Vis. Sci. 45, 764-768 (2004)

24. Li, G. et al. Nonredundant role of Akt2 for neuroprotection of rod photoreceptor cells from light-induced cell death. J. Neurosci. 27, 203-211 (2007).

25. Li, G., Rajala, A., Wiechmann, A. F., Anderson, R. E. \& Rajala, R. V. Activation and membrane binding of retinal protein kinase Balpha/Akt1 is regulated through light-dependent generation of phosphoinositides. J. Neurochem. 107, 1382-1397 (2008)

26. Le, Y. Z. et al. Mouse opsin promoter-directed Cre recombinase expression in transgenic mice. Mol. Vis. 12, 389-398 (2006).

27. Gershon, T. R. et al. Hexokinase-2-mediated aerobic glycolysis is integral to cerebellar neurogenesis and pathogenesis of medulloblastoma. Cancer Metab. 1, 2 (2013).

28. Petit, L. et al. Aerobic glycolysis is essential for normal rod function and controls secondary cone death in retinitis pigmentosa. Cell Rep. 23, 2629-2642 (2018).

29. Rueda, E. M. et al. The cellular and compartmental profile of mouse retinal glycolysis, tricarboxylic acid cycle, oxidative phosphorylation, and $\sim P$ transferring kinases. Mol. Vis. 22, 847-885 (2016).

30. Besirli, C. G., Chinskey, N. D., Zheng, Q. D. \& Zacks, D. N. Autophagy activation in the injured photoreceptor inhibits fas-mediated apoptosis. Invest Ophthalmol. Vis. Sci. 52, 4193-4199 (2011). 
31. Besirli, C. G., Chinskey, N. D., Zheng, Q. D. \& Zacks, D. N. Inhibition of retinal detachment-induced apoptosis in photoreceptors by a small peptide inhibitor of the fas receptor. Invest Ophthalmol. Vis. Sci. 51, 2177-2184 (2010).

32. Besirli, C. G., Zheng, Q. D., Reed, D. M. \& Zacks, D. N. ERK-mediated activation of Fas apoptotic inhibitory molecule 2 (Faim2) prevents apoptosis of $661 \mathrm{~W}$ cells in a model of detachment-induced photoreceptor cell death. PLOS ONE 7 e46664 (2012)

33. Zacks, D. N., Han, Y., Zeng, Y. \& Swaroop, A. Activation of signaling pathways and stress-response genes in an experimental model of retinal detachment. Invest Ophthalmol. Vis. Sci. 47, 1691-1695 (2006).

34. Zacks, D. N. et al. Caspase activation in an experimental model of retinal detachment. Invest Ophthalmol. Vis. Sci. 44, 1262-1267 (2003).

35. Zacks, D. N., Zheng, Q. D., Han, Y., Bakhru, R. \& Miller, J. W. FAS-mediated apoptosis and its relation to intrinsic pathway activation in an experimental model of retinal detachment. Invest. Ophthalmol. Vis. Sci. 45 4563-4569 (2004).

36. Hisatomi, T. et al. Critical role of photoreceptor apoptosis in functional damage after retinal detachment. Curr. Eye Res. 24, 161-172 (2002).

37. Hurley, J. B., Lindsay, K. J. \& Du, J. Glucose, lactate, and shuttling of metabolites in vertebrate retinas. J. Neurosci. Res 93, 1079-1092 (2015).

38. Anderson, M., Marayati, R., Moffitt, R. \& Yeh, J. J. Hexokinase 2 promotes tumor growth and metastasis by regulating lactate production in pancreatic cancer. Oncotarget 8, 56081-56094 (2017).

39. Wolf, A. et al. Hexokinase 2 is a key mediator of aerobic glycolysis and promotes tumor growth in human glioblastoma multiforme. J. Exp. Med. 208, 313-326 (2011).

40. Wilson, J. E. Hexokinases. Rev. Physiol. Biochem Pharm. 126, 65-198 (1995).

41. Chinchore, Y., Begaj, T., Wu, D., Drokhlyansky, E. \& Cepko, C. L. Glycolytic reliance promotes anabolism in photoreceptors. Elife 6, e25946 (2017).

42. Liu, G. M. \& Zhang, Y. M. Targeting FBPase is an emerging novel approach for cancer therapy. Cancer Cell Int. 18, 36 (2018)

43. Wilson, J. E. Isozymes of mammalian hexokinase: structure, subcellular localization and metabolic function. J. Exp. Biol. 206, 2049-2057 (2003).

44. Abu-Hamad, S., Zaid, H., Israelson, A., Nahon, E. \& Shoshan-Barmatz, V. Hexokinase-l protection against apoptotic cell death is mediated via interaction with the voltage-dependent anion channel-1: mapping the site of binding. J. Biol. Chem. 283, 13482-13490 (2008).

45. Schindler, A. \& Foley, E. Hexokinase 1 blocks apoptotic signals at the mitochondria. Cell. Signal. 25, 2685-2692 (2013).

46. Shoshan-Barmatz, V., Zakar, M., Rosenthal, K. \& Abu-Hamad, S. Key regions of VDAC1 functioning in apoptosis induction and regulation by hexokinase. Biochim. Biophys. Acta 1787, 421-430 (2009).

47. Jost, P. J. et al. XIAP discriminates between type I and type II FAS-induced apoptosis. Nature 460, 1035-1039 (2009).

48. O'Driscoll, C., Donovan, M. \& Cotter, T. G. Analysis of apoptotic and survival mediators in the early post-natal and mature retina. Exp. Eye Res. 83 1482-1492 (2006).

49. Green, D. R. \& Llambi, F. Cell death signaling. Cold Spring Harb. Perspect. Biol. 7, a006080 (2015).

50. Zadro-Lamoureux, L. A. et al. XIAP effects on retinal detachment-induced photoreceptor apoptosis [corrected]. Invest. Ophthalmol. Vis. Sci. 50, 1448-1453 (2009).

51. Chang, B. et al. Retinal degeneration mutants in the mouse. Vis. Res. 42, 517-525 (2002)

52. Parapuram, S. K. et al. Distinct signature of altered homeostasis in aging rod photoreceptors: implications for retinal diseases. PLOS ONE 5, e13885 (2010).

53. Wang, A. L., Lukas, T. J., Yuan, M. \& Neufeld, A. H. Age-related increase in mitochondrial DNA damage and loss of DNA repair capacity in the neura retina. Neurobiol. Aging 31, 2002-2010 (2010).

54. Winkler, B. S. The electroretinogram of the isolated rat retina. Vis. Res. 12 1183-1198 (1972)

55. al-Ubaidi, M. R. et al. Bilateral retinal and brain tumors in transgenic mice expressing simian virus 40 large $T$ antigen under control of the human interphotoreceptor retinoid-binding protein promoter. J. Cell Biol. 119, 1681-1687 (1992)

56. Pawar, M. et al. FAS apoptotic inhibitory molecule 2 is a stress-induced intrinsic neuroprotective factor in the retina. Cell Death Differ. 24, 1799-1810 (2017).

57. Li, Y., Fariss, R. N., Qian, J. W., Cohen, E. D. \& Qian, H. Light-induced thickening of photoreceptor outer segment layer detected by ultra-high resolution OCT imaging. Invest Ophthalmol. Vis. Sci. 57, OCT105-OCT111 (2016). 